\title{
Engineering slow light and mode crossover in a fractal-kagomé waveguide network
}

\author{
Atanu Nandy and Arunava Chakrabart \\ Department of Physics, University of Kalyani, Kalyani, West Bengal-741235, India
}

\begin{abstract}
We present an analytically exact scheme of unraveling a multitude of flat, dispersionless photonic bands in a kagomé waveguide strip where each elementary plaquette hosts a deterministic fractal geometry of arbitrary size. The number of non-dispersive eigenmodes grows as higher and higher order fractal geometry is embedded in the kagomé motif. Such eigenmodes are found to be localized with finite support in the kagomé strip and exhibit a hierarchy of localization areas. The onset of localization can, in principle, be delayed in space by an appropriate choice of frequency of the incident wave. The length scale at which the onset of localization for each mode occurs, can be tuned at will as prescribed here using a real space renormalization method. Conventional methods of extracting the non-dispersive modes in such geometrically frustrated lattices fail as a non-translationally invariant fractal decorates the unit cells in the transverse direction. The scheme presented here circumvents this difficulty, and thus may inspire the experimentalists to design similar fractal incorporated kagomé or Lieb class of lattices to observe a multifractal distribution of flat photonic bands.
\end{abstract}

PACS numbers: 63.20.Pw, 42.25.Bs, 42.25.Kb, 42.82.Et

\section{INTRODUCTION}

The geometrically frustrated lattices (GFL) have shown growing interest over the past few years not only for the emergence of exotic spin liquid, spin ice and charge and magnetic disordered phases in strongly correlated systems [1]-[5], but also very recently for discerning the existence of flat non-dispersive electronic states leading to remarkable band structures in a wide variety of physical systems [6]- 9] , as well as in several artificial model lattices [10, 11]. The topology of the underlying lattice and destructive quantum interference play a pivotal role in localizing single particle eigenstates even when there is no apparent disorder in the system. The eigenstates have non-zero amplitudes either on specific atomic sites, or over clusters of sites which are effectively separated from their neighboring clusters by sites on which the amplitude of the wave function vanishes. This results in non-dispersive energy-wave vector relationship which makes the effective mass of an electron diverging, leading to an complete or extremely low mobilty of the particle [10, 11].

As is well known now, localization is common to classical waves as well, including light. The concept of photonic band gap (PBG) was initiated by Yablonovitch [12, 13] along with the proposals from John [14] and Pendry and MacKinon [15] regarding the possibility of observing the Anderson localization of light. Recent direct experimental observation of localized photonic flat band states [16, 17] in Lieb photonic lattices and tight binding photonic bands in metallophotonic waveguide networks [18] have enriched and stimulated research in photonics. Such 'flat' photonic bands are intimately connected to the pathbreaking idea of engineering slow light

*Electronic address: atanunandy1989@gmail.com

${ }^{\dagger}$ Electronic address: arunava chakrabarti@yahoo.co.in with low group velocity which opens up the possibility of a 'spatial compression of light energy' and the related linear and non linear optical effects [19]. A new exciting area in photonics is thus very much on the cards.

Inspired by these experiments and other works on localization of light in tailor made lattices, in the present communication we address the problem of localization of electromagnetic waves and a controlled engineering of the flat, non dispersive photonic bands in a kagomé strip, made of single mode waveguides. In this work each elementary unit cell of the kagomé geometry encloses a Sierpinski gasket (SPG) 20] network made of similar single mode waveguides, and of arbitrarily large generation ( Fig:1(a)). This construction introduces a non trivial modification of the existing waveguide networks in the kagomé class, and throws an achievable challenge to the experimentalists in this era of pretty advanced nanotechnology and lithography techniques.

The principal motivation behind this idea is to look into the possibility of generating a whole hierarchy of non-dispersive, flat optical bands, if they exist, and to work out a scheme for locating them in the optical spectrum of the kagomé-fractal system. An SPG network, in the context of electrons, is already known to possess an infinite number of extended, totally transmitting eigenstates even in the absence of any long range translational order. Such states densely populate the otherwise Cantor set energy spectrum consisting of localized eigenfunctions [21]. This is likely to be the case for optical modes as well. If it is true, then the proximity of sharply localized non-dispersive modes to the totally transmitting (extended), dispersive ones (as the fractal grows in size) may lead to a new interesting scenario where a comprehensive control can be achieved over a possible transition between slow and resonant optical modes as a function of the frequency of the light injected into the waveguide network.

It should also be appreciated that, while a kagomé geometry in its simplest shape, is already known to ex- 
hibit flat photonic bands, the incorporation of the fractal geometry in each unit cell destroys the translational order locally (though it is preserved on a global scale in the horizontal direction) in the transverse direction. This makes the conventional methods of extracting flat band states impossible to be implemented, specially as the gasket grows in size. Straightforward diagonalization of hamiltonian (for electronic case) or an exact numerical solution of the homogeneous wave equation may not help because of highly fragmented spectral character, and the eigenmodes obtained from a finite size system may slip away from the original spectrum as we move over to a higher generation. We prescribe a simple method to circumvent this problem by exploiting the scale invariance of the gasket structure and a real space renormalization group (RSRG) method. The method can be applied practically to any quasi-one dimensional systems and, to the best of our knowledge, has not been reported before as far as the evaluation of flat photonic bands are concerned.

The other point of interest in the present work is to analyze the distribution of the amplitudes of such flat band states in real space. Recently, a class of localized eigenfunctions in deterministic fractal geometries has been revealed both in the context of electronic states and optical modes [22, 23] which can in principle, be delayed in space by an appropriate choice of the energy of the electron or the frequency of the injected wave. Such states exhibit a fragmented distribution in the respective spectra, a consequence of the underlying lattice topology. We would like to examine whether such states, if they exist in the present network, can be discerned inspite of the complicated geometry of the network, and that, if they really correspond to the non dispersive, flat character which has been of immense interest recently.

Before we end this section, it is worth mentioning that, single mode waveguides have recently been fabricated and used in a quasiperiodic optical setup [24] to unravel topological states in quasicrystals. Localization transition in one dimensional quasiperiodic lattices has also been investigated experimentally in recent times 25]. The present proposal, to our mind, can thus be tested with an appropriately designed waveguide network.

In what follows we describe our findings. In section II we describe the wave equation and the RSRG scheme. Section [II contains a discussion regarding the density of eigenmodes, existence of non-dispersive eigenmodes, and the dispersion relation. Finally, in section IV we draw our conclusions.

\section{THE WAVE EQUATION AND THE RSRG SCHEME}

\section{A. The difference equations}

Let us begin by refering to Fig. 1(a), where we have taken a typical waveguide network formed by waveguide segments having the same dimensions arranged in a kagomé geometry. A Sierpinski gasket fractal network of finite generation is embedded inside the skeleton of kagomé geometry within each 'unit cell'. Each segment has a single channel for wave propagation. The wave propagation inside the core of the waveguide is governed by the wave equation [26, 27], viz.,

$$
\frac{\partial^{2} \psi_{i j}(x)}{\partial x^{2}}+k^{2} \psi_{i j}(x)=0
$$

where, $\psi_{i j}(x)$ is the photonic wavefunction between two nodes $i$ and $j$, and $k=\frac{\omega \sqrt{\epsilon_{r}}}{c_{0}}$ is the wave vector inside the material of the waveguide. $\omega$ is the frequency of the wave

(a)

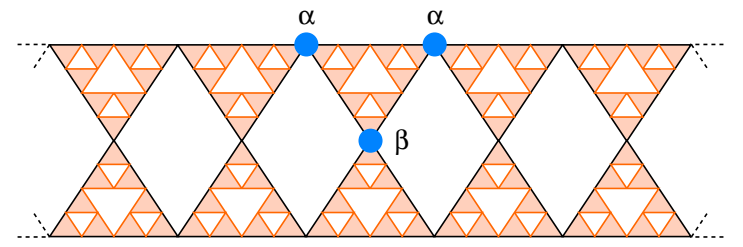

(b)

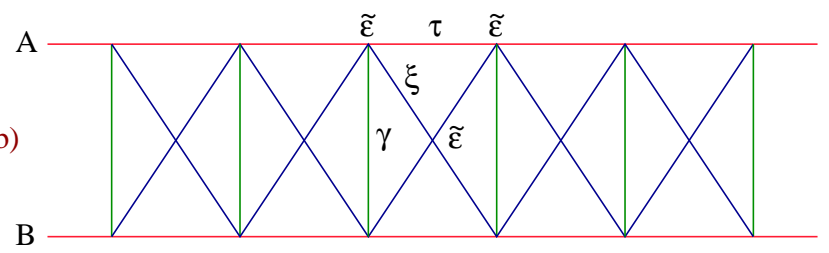

FIG. 1: (Color online) (a) An infinite fractal incorporated kagomé waveguide network and (b) the effective renormalized ladder network.

propagating, $c_{0}$ is the speed of light in free space and $\epsilon_{r}$ contains all the dielectric information of the material of the core of the waveguide. The above Eq. (10) has the obvious solution of the form [26, 27],

$$
\psi_{i j}(x)=\psi_{i} \frac{\sin \left[k\left(l_{i j}-x\right)\right]}{\sin k l_{i j}}+\psi_{j} \frac{\sin k x}{\sin k l_{i j}}
$$

$x$ is the variable distance measured from $i$-th nodal point, $l_{i j}$ is the length of the waveguide segment between $i$-th and $j$-th node and $\psi_{i}$ and $\psi_{j}$ characterizes the wavefunction at the corresponding nodes respectively. The flux conservation condition yields,

$$
\sum_{j}\left[\frac{\partial}{\partial x} \psi_{i j}(x)\right]=0
$$

where the summation $j$ runs over all possible nodes connected directly to $i$, and this finally leads to a discretized version of Eq. (1), viz.,

$$
-\psi_{i} \sum_{j} \cot \theta_{i j}+\sum_{j} \psi_{j} / \sin \theta_{i j}=0
$$

where $\theta_{i j}=k l_{i j}=k a, a$ being the constant waveguide dimension. The above Eq. (4) is an exact replica of the equation for spinless non-interacting electrons propagating in a lattice. The difference equation, an equivalent 
discretized form of Schrödinger's equation in this latter case reads,

$$
\left(E-\epsilon_{i}\right) \psi_{i}=\sum_{j} t_{i j} \psi_{j}
$$

Here, $E$ is the electron energy, $\epsilon_{i}$ is the onsite potential at each atomic site, and $t_{i j}$, the nearest neighbor hopping integral, signifying the overlap of electronic wavefunctions between two neighboring sites.

We exploit this resemblance to analyse the existence of dispersionless flat photonic bands as well as the extended Bloch-like eigenmodes in such typical waveguide system. It is trivial to observe that, a replacement of $k a$ by $\pi-$ $k a$ yields new recast version of Eq. (5) with $t_{i j}$ being replaced by $-t_{i j}$ which does not affect the spectrum in any way.

The one-to-one correspondence of Eq. (4) and Eq. (5), prompts us to identify $E-\epsilon_{i}=-\sum_{j} \cot \theta_{i j}$ and $t=$ $1 / \sin k a$. It is then simple to extract information about the photonic modes using the language of the electrons, and the results will be exact. As illustrated in Fig. 1, the fractal-kagomé waveguide network contains nodes with four nearest neighbors only. Therefore, for classical wave propagation we just assign $E-\epsilon_{i}=-4 \cot k a$ for every vertex ' $i$ '. The overlap integral along the arm of each basic triangular plaquette is $t=1 / \sin k a$ carries the kinetic information of the propagating wave.

Using a real space renormalization group method, we now proceed to describe our basic scheme and the physics of wave propagation in such fractal incorporated kagomé geometry by exploiting the exact analogy with the corresponding electronic problem.

\section{B. Converting an SPG-kagomé network into a two-strand ladder}

Let us suppose that an SPG at its $n$th generation is grafted inside every unit cell of the kagomé strip
(Fig. 1(a)). As proposed before, we set $\epsilon_{i}=E+$ $4 \cot k a=\epsilon$ at each node and $t=1 / \sin k a\left(k a=\theta_{i j}\right.$ is assumed constant, as we take all the waveguide segments to be identical in every respect). $E$ can be chosen quite arbitrarily (playing the role of Fermi energy in the equivalent electronic case), and this selection in the present case sets the range of frequency of the injected wave.

With this assumption, we decimate out a subset of the wave amplitudes on the bulk vertices of the SPG fractal system in terms of others surviving vertices to generate an effective 'two-arm ladder' (Fig. 1(b)). This ladder is now characterized by the renormalized values of the onsite potentials at its vertices, $\tilde{\epsilon}=\epsilon_{n}+2 t_{n}^{2} /\left(E-\epsilon_{n}\right)$, the hopping integral along each arm $\tau=t_{n}+t_{n}^{2} /\left(E-\epsilon_{n}\right)$, the inter-arm (between arms $A$ and $B$ ) hopping integral $\gamma=2 t_{n}^{2} /\left(E-\epsilon_{n}\right)$, and the second neighbor hopping inside every plaquette $\xi=t_{n}^{2} /\left(E-\epsilon_{n}\right)$. This latter quantity is a result of the decimation of the 'internal' vertices in a unit cell.

The quantities $\epsilon_{n}$, and $t_{n}$ are obtained following the usual RSRG recursion relations in the analogous electronic case [21], viz.,

$$
\begin{aligned}
\epsilon_{n} & =\epsilon_{n-1}+\frac{4 t_{n-1}^{2}\left(E-\epsilon_{n-1}\right)}{\left(E-\epsilon_{n-1}\right)^{2}-\left(E-\epsilon_{n-1}\right) t_{n-1}-2 t_{n-1}^{2}} \\
t_{n} & =\frac{2 t_{n-1}^{3}+\left(E-\epsilon_{n-1}\right) t_{n-1}^{2}}{\left(E-\epsilon_{n-1}\right)^{2}-\left(E-\epsilon_{n-1}\right) t_{n-1}-2 t_{n-1}^{2}}
\end{aligned}
$$

with $\epsilon_{0}=\epsilon$ and $t_{0}=t$. The difference equations for the ladder network now read,

$$
\begin{aligned}
(E-\tilde{\epsilon}) \psi_{n, A} & =\tau\left(\psi_{n+1, A}+\psi_{n-1, A}\right)+\gamma \psi_{n, B}+\xi\left(\psi_{n+1, B}+\psi_{n-1, B}\right) \\
(E-\tilde{\epsilon}) \psi_{n, B} & =\tau\left(\psi_{n+1, B}+\psi_{n-1, B}\right)+\gamma \psi_{n, A}+\xi\left(\psi_{n+1, A}+\psi_{n-1, A}\right)
\end{aligned}
$$

The Eq. (7) can be written in an equivalent matrix form, viz.,

$$
\left[\left(\begin{array}{cc}
E & 0 \\
0 & E
\end{array}\right)-\left(\begin{array}{cc}
\tilde{\epsilon} & \gamma \\
\gamma & \tilde{\epsilon}
\end{array}\right)\right]\left(\begin{array}{l}
\psi_{n, A} \\
\psi_{n, B}
\end{array}\right)=\left(\begin{array}{cc}
\tau & \xi \\
\xi & \tau
\end{array}\right)\left(\begin{array}{l}
\psi_{n+1, A} \\
\psi_{n+1, B}
\end{array}\right)+\left(\begin{array}{cc}
\tau & \xi \\
\xi & \tau
\end{array}\right)\left(\begin{array}{l}
\psi_{n-1, A} \\
\psi_{n-1, B}
\end{array}\right)
$$


This means they can be diagonalized simultaneously using a similarity transform. Taking advantage of this, we make a uniform change of basis, going from the ba$\operatorname{sis} \boldsymbol{\Psi}_{\mathbf{n}} \equiv\left(\psi_{\mathbf{n}, \mathbf{A}}, \psi_{\mathbf{n}, \mathbf{B}}\right)$ to a new basis $\boldsymbol{\Phi}_{\mathbf{n}} \equiv\left(\phi_{\mathbf{n}, \mathbf{A}}, \phi_{\mathbf{n}, \mathbf{B}}\right)$, such that, $\boldsymbol{\Phi}_{\mathbf{n}}=\mathbf{M}^{-1} \mathbf{\Psi}_{\mathbf{n}}$. The matrix $\mathbf{M}$ diagonalizes both the 'potential' and the 'hopping' matrices. This leads to a decoupling of the coupled set of equations Eq. (77) 28, 29]. The decoupled equantions read,

$$
\begin{aligned}
{\left[E-\left(\epsilon_{n}-\frac{4 t_{n}^{2}}{E-\epsilon_{n}}\right)\right] \phi_{n, A} } & =\left(t_{n}+\frac{2 t_{n}^{2}}{E-\epsilon_{n}}\right)\left(\phi_{n+1, A}+\phi_{n-1, A}\right) \\
\left(E-\epsilon_{n}\right) \phi_{n, B} & =t_{n}\left(\phi_{n+1, B}+\phi_{n-1, B}\right)
\end{aligned}
$$

The decoupled equations individually represent two independent linear chains along which some quantum particle propagates, and its spectrum will be identical to that of the photonic waveguide we have constructed, as the equivalence between the two cases is an exact one.

We now proceed to explore these equations to unravel the dispersionless modes, if any, and the others for the kagomé fractal waveguide.

\section{RESULTS AND DISCUSSION}

\section{A. The general character of the spectrum}

Before looking for the flat photonic bands and the extended eigenmodes, it is advisable to have a general idea of the spectrum of allowed photon frequencies in such a system. It should be appreciated that, each of the decoupled equations in Eq. (77) represents a purely ordered chain of identical 'atomic' sites with effective, energy dependent potentials and hopping integrals. Each equation has its own density of states spectrum, and a convolution of these will give the true density of states of the actual system. The fragmentation of the spectra and their subband structures will depend on the generation of the embedded SPG network.

We have however, used an RSRG decimation scheme on the kagomé fractal in Fig. 1(a) to work out the local Green's function $G_{00}=G_{00}^{\alpha}+G_{00}^{\beta}$. The superscripts $\alpha$ and $\beta$ refer to the top and middle vertices in the kagomé skeleton, as marked by solid blue circles in Fig. 1(a). We refrain from including the detailed mathematical expressions exploited to arrive at the result just to save space, and provide a typical local density of photonic modes (LDPM) as a function of normalized frequency when first and second generation SPG fractals are inserted in an infinite kagomé strip is shown in Fig. 2, as obtained from the well known expression,

$$
\rho(\omega)=(-1 / \pi) \lim _{\eta \rightarrow 0} \operatorname{Im} G_{00}(\omega+i \eta)
$$

The distribution of eigenmodes plotted within $0<$ $\omega / 2 \pi c<1 / 2$ shows clusters of nonzero values of LDPM over finite range of $\omega$. Quite arbitrarily we have set
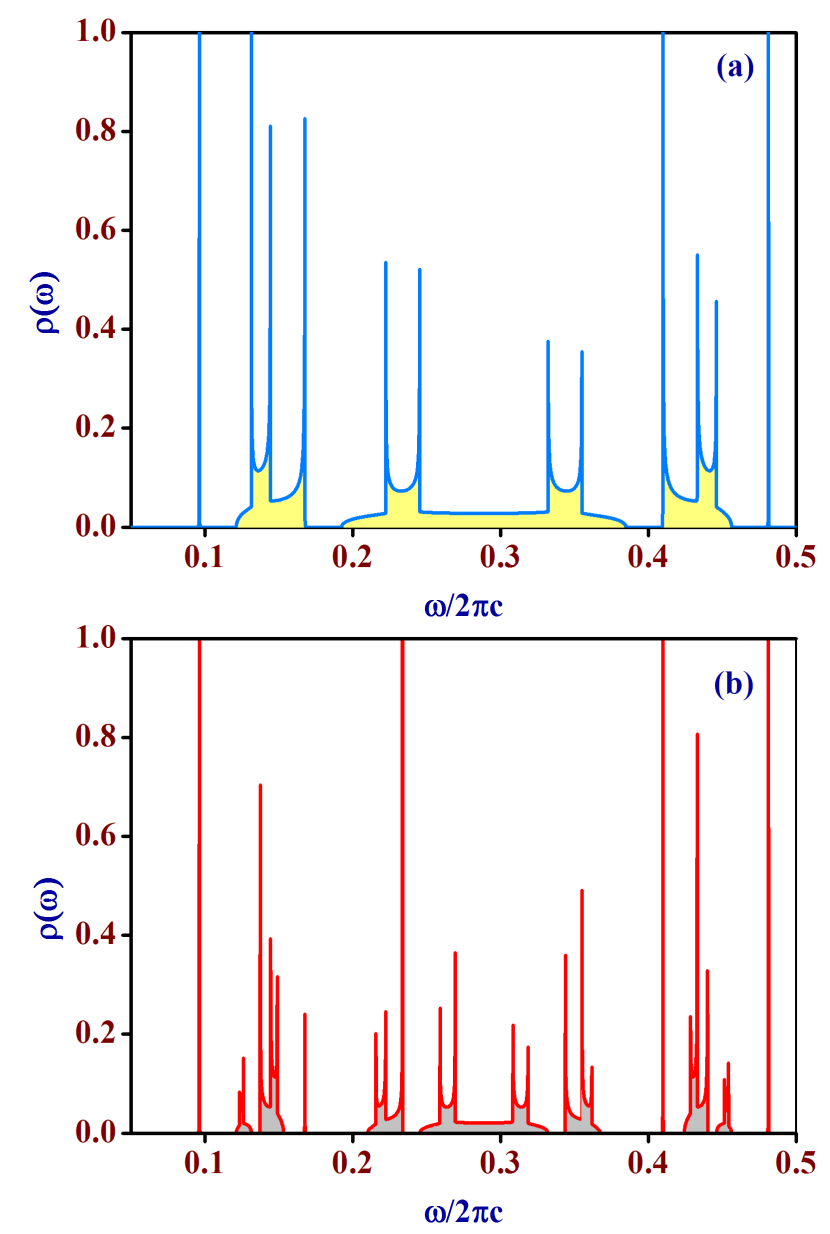

FIG. 2: (Color online) Density of eigenmodes as a function of normalized frequency for a fractal-kagomé waveguide network when an SPG geometry of (a) $1^{\text {st }}$ generation and (b) $2^{\text {nd }}$ generation is embedded into it.

$\epsilon_{r}=3$. The network exhibits gaps in the frequency spectrum in which the propagation of wave is not allowed and thus is a model PBG system. With increasing generations of the embedded SPG fractal the LDPM gradually assumes a fragmented Cantor-like character, the key signature of the fractal itself that fills up a kagomé unit cell. The signature is already apparent in Fig. 2 


\section{B. The Non-dispersive eigenmodes}

If a single photonic mode is dispersionless in one direction, it implies localization in that direction as an extreme case, unfolding the possibility of engineering ultra slow light. This results from a perfect geometric phase cancellation which causes an element of the effective mass tensor to be divergent, leading to a possible 'observation' of heavy photons. This doesn't look improbable if we borrow the language for the electrons, thanks to the analogous tight binding model which indeed works quite accurately for the low lying photonic dispersion. Flatness in the frequency $(\omega)$-vs-wave vector $(q)$ curves implies infinite effective mass of the particle involved. This in turn, makes the group velocity vanish. The kinetic energy of the associates wave packet is quenched, and the density of states diverges due the relationship

$$
\rho_{\omega} \propto \int v_{g}^{-1} d^{3} q
$$

where, $v_{g} \propto d \omega / d q[\underline{30}]$. Such singularities in the density of states are expected to produce anomalous behaviors in physical properties as well as transport phenomena and optical response.

Because of zero group velocity, the corresponding single particle state is sharply localized at a point, or in a finite cluster of nodal points in the system. Such clusters are separated from the neighboring clusters by vertices where the amplitude of the wave function is zero. Therefore, the movement of the particle is restricted along the periphery of those finite clusters. The important question is, how can we extract such eigenvalues for a kagoméfractal network?

In the first of decoupled set of equations Eq. (7), if we set $t_{n}+2 t_{n}^{2} /\left(E-\epsilon_{n}\right)=0$, we get $E=\epsilon_{n}-2 t_{n}$. The first equation now represents, in the language of the tight binding scheme, the difference equation of an isolated (effective) 'atom'. The corresponding eigenstate(s) will be sharply localized, provided one gets non-zero density of states at these special energies. These will be the desired non dispersive, flat band (FB) modes, as will be explicit towards the end of this subsection. With $\epsilon_{r}=3$, the frequencies of the 'flat band mode' turn out to be $\omega / 2 \pi c=0.096225,0.167561,0.409795$, and 0.48113 . These correspond to the isolated spike at the left end of Fig. 2(a), the right and the left 'edges' of the first and third subbands (yellow shaded) respectively, and the sharply localized mode at extreme right. The roots corresponding to the edges (0.167561 and 0.409795$)$ separate out of the bands when the second generation SPG is inserted into the unit cell of the kagomé strip, as seen from Fig. 2(b). Obviously, as we extract the roots from the deeper and deeper scales of length (which means sequentially setting $n=1,2,3, \ldots .$.$) , more and more such$ dispersionless FB states result. Every FB mode arising out of a given generation $n$ and forming the edge of a subband gets detached from it and stand out separate for all subsequent generations $n+m$, with $m=1,2,3$, ........

For an electromagnetic wave with an arbitrary wave vector $q$ injected in the SPG-kagomé network, each of the two decoupled equations Eq. (7), can be simultaneously used in analogy with the dynamics of an electron in a periodic array of (effective) potentials, to obtain the

(a)

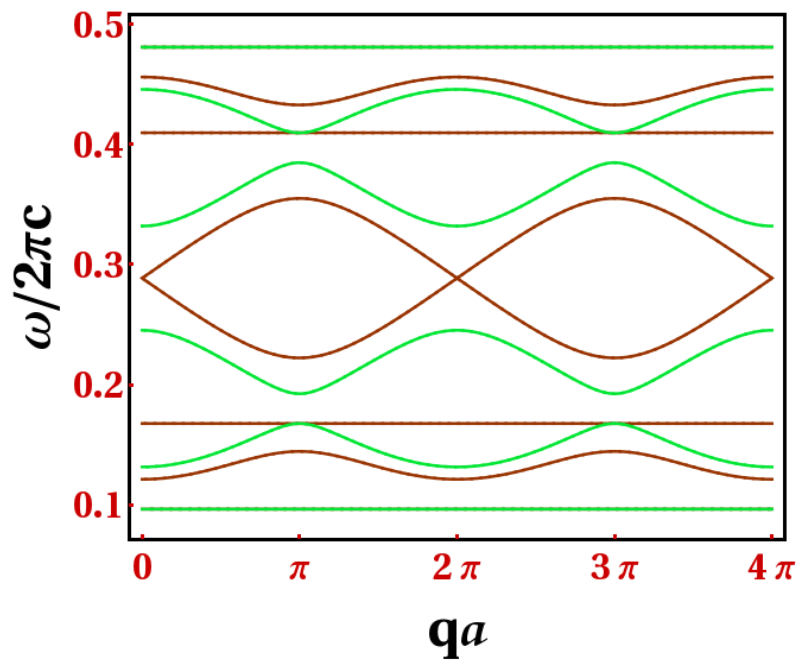

(b)

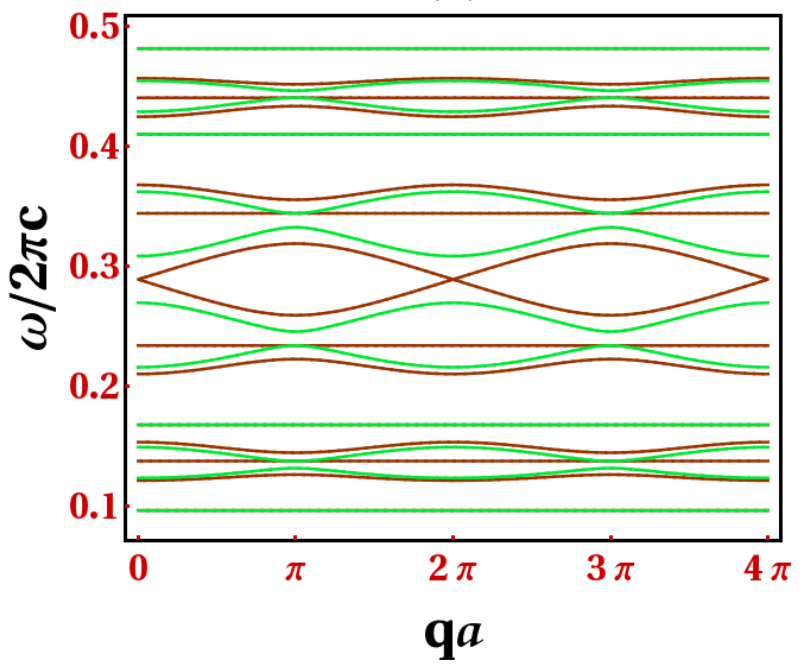

FIG. 3: (Color online) Dispersion relation for a fractal-kagomé waveguide network when a $b=2$ SPG geometry of (a) $1^{\text {st }}$ generation and (b) $2^{\text {nd }}$ generation is embedded into it. The green and brown lines correspond to solutions obtained from the first and the second decoupled equations respectively.

dispersion relation for photons. This obviously displays the $\omega / 2 \pi c-q a$ curves, bringing out the 'allowed' photonic bands as functions of the injected wave vector. The photonic dispersion relations, for materials with $\epsilon_{r}=3$, and when a $1^{\text {st }}$ generation SPG is inserted in the kagomé motif read, 


$$
\begin{aligned}
{\left[2 \cos \left(2 \pi \sqrt{\epsilon_{r}} \omega^{\prime} a\right)-1\right]\left[4 \cos \left(2 \pi \sqrt{\epsilon_{r}} \omega^{\prime} a\right)+1\right]\left[6 \cos \left(2 \pi \sqrt{\epsilon_{r}} \omega^{\prime} a\right)+4 \cos \left(4 \pi \sqrt{\epsilon_{r}} \omega^{\prime} a\right)+3-\cos q a\right] } & =0 \\
{\left[6 \cos \left(2 \pi \sqrt{\epsilon_{r}} \omega^{\prime} a\right)+4 \cos \left(4 \pi \sqrt{\epsilon_{r}} \omega^{\prime} a\right)+4-\cos q a\right]\left(2 \cos \left(2 \pi \sqrt{\epsilon_{r}} \omega^{\prime} a\right)-1\right) } & =0
\end{aligned}
$$

In the above Eq. (12) $\omega^{\prime}=\omega / 2 \pi c$. The flat, dispersionless modes are easily extracted by setting $\left[4 \cos \left(2 \pi \sqrt{\epsilon} \omega^{\prime} a\right)+1\right]=0$, and, $\left[2 \cos \left(2 \pi \sqrt{\epsilon} \omega^{\prime} a\right)-1\right]=0$ respectively. The roots match exactly with the values of the normalized frequency $\omega / 2 \pi c$ for the sharply localized, atomic like states, as evaluated earlier.

In the top panel of Fig. 3(a) we show the roots of Eq. (12) when a $1^{\text {st }}$ generation SPG is embedded in the unit cells of a kagomé strip. Completely flat frequency $(\omega / 2 \pi c)$ versus wave vector $(q)$ are apparent. The lower panel exhibits similar flat bands, now more in number as a higher $\left(2^{\text {nd }}\right)$ generation SPG network is grafted in the kagomé strip.

It is interesting to work out that the roots obtained as solutions of the equation $E-\epsilon_{n}+2 t_{n}=0$ are also solutions of the equations $E-\epsilon_{n+1}+2 t_{n+1}=0$. That is, the flat band eigenmodes exhibit a nested pattern. This is also explicitly seen from the figures displayed in Fig. 3. Interestingly, the outermost flat bands remain 'undisturbed', while the neighborhood of the inner ones get densely packed with dispersive and non dispersive modes as one increases the generation of the embedded SPG.

\section{The extended eigenmodes and the crossover}

The similarity of the wave equation and the tight binding one for the electrons help us extract an infinite number of extended Bloch-like eigenmodes in the kagoméfractal network. This is simply done as one observes, using Eq. (6) that once we fix $E=\epsilon_{n}$ at any $n$-th stage of renormalization, the 'hopping integral' gets locked into a 2 -cycle fixed point, viz., $t_{n+2}=-t_{n+1}=t_{n}$. A non-zero value of the hopping integral at all stages of renormalization implies finite overlap of the 'wavefunction' at all scales of length - a clear signature of the corresponding mode being extended (a transparent state, in terms of transmission). For example, if we set $E=\epsilon_{1}$, the principal values of the resulting frequencies corresponding to the extended photonic mode for waveguide materials with $\epsilon_{r}=3$ turn out to be $\omega / 2 \pi c=1 / 4 \sqrt{3}, \sqrt{3} / 4$. Out of these, the first root is a solution of the equation $E=\epsilon_{0}$ at the bare length scale. The Eq. (6) exhibits a nesting pattern of the roots in the sense that, the solutions of the equation $E-\epsilon_{n}=0$ includes the solutions already obtained from the equation $E-\epsilon_{n-1}=0$.

As one should now be able to appreciate that, as one inserts larger and larger versions of the SPG inside a kagomé unit cell, both the flat band eigenmodes and the extended ones get densly packed in the dispersion curves (as well as in the LDPM graphs). The curvatures of even the dispersive modes start becoming lesser and lesser indicating a lowering of the group velocity. The dense packing of the flat and the dispersive bands therefore opens up a possibility of engineering the so called slow light and re-entrant crossover of optical eigenmodes, going from an extended (dispersive) to a sharply localized (flat) one, or vice versa as one climbs the frequency axis at any fixed value of the wave vector $q$. Such a crossover can, in principle, be controlled over arbitrarily small intervals of energy, thanks to the intrinsic multifractal character of the energy spectrum of the embedded SPG geometry.

\section{Spatial distribution of the flat band states and the staggered localization effect}

The localized character of the eigenmodes are illustrated in Fig. 4, where the amplitudes of the photonic

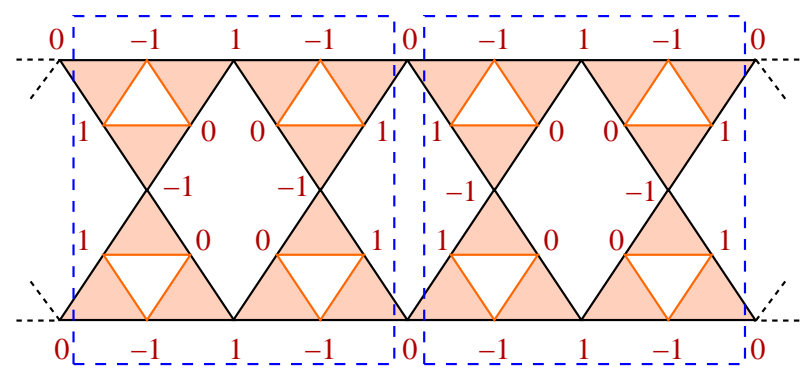

FIG. 4: (Color online) Distribution of amplitude for the flat non-dispersive photonic state at $\omega=0.096225$ (in the unit of $2 \pi c$ ) for the fractal incorporated kagomé waveguide network. The blue dotted zones are the allowed zones getting decoupled from each other by the vertices having zero amplitude of the wavefunctions.

modes are shown only at the vertices of the kagomé motif for a clearer understanding. The 'spanning' plaquettes, in which the amplitudes are mostly confined are shaded for identification. Fig. 4 brings out distribution corresponding to the basic flat band frequency $\omega / 2 \pi c=0.096225$. One blue dotted square is 'separated' from its neighboring squares by a couple of sites on which the amplitudes disappear. For a frequency extracted from a higher generation $n$, the amplitudes spread over larger clusters, but one such cluster remains decoupled from its neighbors through vertices at which the amplitude of the excitation vanishes.

It is encouraging to extend this concept when increasing generations of the SPG occupy the plaquettes. One can then extract flat, non-dispersive modes for which the 
distribution of amplitudes will assume a non trivial shape over larger clusters of vertices. Thus, the onset of localization will be felt at a relatively larger scale of length. How large - depends on the scale (or generation index $n$ ) at which we are solving the dispersion equation.

This leads to the concept of staggered localized modes, where one can have the liberty to delay the onset of localization in space using the RSRG index, and an appropriate frequency [23].

The FB states talked about so far are in fact localized around each crossing, with a rapidly decaying tail toward the neighboring junctions. The difference equation corresponding to each vertex can easily be exploited to construct a bound state amplitude configuration such as shown in the Fig. 4. By contrast, resonant states or the dispersive states, which have intrinsic losses, will couple to extended states to result in significant transfers to distant neighbors, deteriorating the tight-binding picture with near-neighbor transfers.

\section{CLOSING REMARKS}

In conclusion, we have critically examined the photonic spectral structure a the kagomé monomode waveguide strip which with embedded Sierpinski gasket fractal mesh in every unit cell. The spectrum is shown to contain a countable infinity of flat, dispersionless optical modes which are localized in clusters of finite size, displaying a multitude of localization area. Such clusters are effec- tively separated from similar clusters in the network by nodes where the amplitude of the wave function is zero. We have been able to present an analytical prescription for the determination of frequency of such non-dispersive eigenmodes. The size of the clusters can be tuned at will by the length scale at which the frequency is evaluated. The onset of localization may be delayed in space (staggered).

Such results give an opportunity to modulate experimentally the localization of classical waves, for example, light, triggered by the lattice topology without bothering about the high permittivity of the core materials. It might be useful in developing novel photonic band-gap structures, where the pass band can be controlled over arbitrarily small domains by exploiting the fractal signature of the network. Also, the physical significance of vanishing group velocity is that several scattering waves form standing wave pattern in such photonic waveguide network. For such self-localized eigenmodes, we can obtain coherent waves, i.e., lasing action at the photonic band edges.

\section{Acknowledgments}

AN acknowledges financial support through a research fellowship [Award letter no. F.17-81/2008 (SA-I)] from UGC, India. AC acknowledges a partial financial support from University of Kalyani through its DST-PURSE grant.
[1] F. Mezzacapo and M. Boninsegni, Phys. Rev. B 85, 060402(R) (2012).

[2] T. Yoshioka, A. Koga, and N. Kawakami, Phys. Rev. Lett. 103, 036401 (2009).

[3] T. Ohashi, N. Kawakami, and H. Tsunetsugu, Phys. Rev. Lett. 97, 066401 (2006).

[4] M. Jarrell and H. R. Krishnamurthy, Phys. Rev. B 63, 125102 (2001).

[5] W. Wu, Y. H. Chen, H. S. Tao, N. H. Tong, and W. M. Liu, Phys. Rev. B 82, 245102 (2010).

[6] L. Zheng, L. Feng, and W. Y.-Shi, Chin. Phys. B 23, 077308 (2014).

[7] O. Derzhko, J. Richter, and M. Maksymenko, Int. J. Mod. Phys. B 29, 1530007 (2015).

[8] H. Wang, J-H. Gao, and F-C. Zhang, Phys. Rev. B 87, 255116 (2013).

[9] O. Derzhko, J. Richter, A. Honecker, M. Maksymenko, and R. Moessner, Phys. Rev. B 81, 014421 (2010).

[10] A. Nandy, B. Pal, and A. Chakrabarti, J. Phys.: Condens. Matter 27, 125501 (2015).

[11] A. Nandy and A. Chakrabarti, Phys. Lett. A 379, 2876 (2015).

[12] E. Yablonovitch, Phys. Rev. Lett. 58, 2059 (1987).

[13] E. Yablonovitch, T. J. Gmitter, and K. M. Leung, Phys. Rev. Lett. 67, 2295 (1991).

[14] S. John, Phys. Rev. Lett. 58, 2486 (1987).

[15] J. B. Pendry and A. MacKinon, Phys. Rev. Lett. 67,
2772 (1992).

[16] S. Mukherjee, A. Spracklen, D. Choudhury, N. Goldman, P. Öhberg, E. Andersson, and R. R. Thomson, Phys. Rev. Lett. 114, 245504 (2015).

[17] R. A. Vicencio, C. Cantillano, L. M-Inostroza, B. Real, S. Weimann, A. Szameit, and M. I. Molina, Phys. Rev. Lett. 114, 245503 (2015).

[18] S. Endo, T. Oka, and H. Aoki, Phys. Rev. B 81, 113104 (2010).

[19] T. Baba, Nature Photonics 2, 465 (2008).

[20] E. Domany, S. Alexander, D. Bensimon, and L. P. Kadanoff, Phys. Rev. B 28, 3110 (1983).

[21] A. Chakrabarti, J. Phys.: Condens. Matter 8, 10951 (1996).

[22] B. Pal and A. Chakrabarti, Phys. Rev. B 85, 214203 (2012).

[23] B. Pal, P. Patra, J. P. Saha, and A. Chakrabarti, Phys. Rev. A 87, 023814 (2013).

[24] Y. E. Kraus, Y. Lahini, Z. Ringel, M. verbin, and O. Zilberberg, Phys. Rev. Lett. 109, 106402 (2012).

[25] Y. Lahini, R. Pugatch, F. Pozzi, M. Sorel, R. Morandotti, N. davidson, and Y. Silberberg, Phys. Rev. Lett. 103, 013901 (2009).

[26] S. Alexander, Phys. Rev. B 27, 1541 (1983).

[27] Z.-Q. Zhang and P. Sheng, Phys. Rev. B 49, 83 (1994).

[28] S. Sil, S. K. Maiti, and A. Chakrabarti, Phys. Rev. Lett. 
101, 076803 (2008).

[29] S. Sil, S. K. Maiti, and A. Chakrabarti, Phys. Rev. B 78, 113103 (2008).
[30] M. Hyrkäs, V. Apaja, and M. Manninen, Phys. Rev. A 87, 023614 (2013). 